\title{
Validation of Next-Generation Sequencing and Array Comparative Genomic Hybridization for Diagnosis and Screening of Reciprocal and Robertsonian Translocations in Human Blastocysts
}

\section{Shengrong Du}

fujian provincial maternity and children's hospital https://orcid.org/0000-0003-0273-6698

Yun-Hong Lin

fujian provincial maternity and children's hospital

Yan Sun

fujian provincial maternity and children's hospital

Qing-Fen Chen

fujian provincial maternity and children's hospital

\section{Zhi-Qing Huang}

fujian provincial maternity and children's hospital

Jing-Qian Su

Fujian Normal University

Bei-Hong Zheng ( $\sim 26921140 @ q q . c o m$ )

\section{Research}

Keywords: array comparative genomic hybridization, next-generation sequencing, reciprocal translocations, Robertsonian translocations

Posted Date: June 25th, 2020

DOl: https://doi.org/10.21203/rs.3.rs-37764/v1

License: (c) (1) This work is licensed under a Creative Commons Attribution 4.0 International License. Read Full License 


\section{Abstract}

Background: Advances in biotechnology, especially next-generation sequencing (NGS) and array comparative genomic hybridization $(\mathrm{aCGH})$ approaches, have improved preimplantation genetic screening; however, these methods have not been directly compared. This study was carried out to identify the more promising method for screening reciprocal and Robertsonian translocations. Here, blastocysts from carriers with reciprocal and Robertsonian translocations were retrospectively evaluated and results from preimplantation genetic testing in 272 blastocytes were analysed for parental unbalanced translocations using aCGH and NGS.

Results: There was no significant difference in the no embryo-transfer rate between aCGH and NGS. Among 59 blastocysts screened in the aCGH group, $32.76 \%$ were normal embryos and $67.24 \%$ were abnormal embryos, including $36.21 \%$ embryos with a translocation, $17.24 \%$ with no translocation, and $15.52 \%$ with combined abnormalities. Similar results were obtained from the 214 blastocysts tested in the NGS group. In women <35-years, more normal blastocysts were identified in the NGS group compared to the aCGH group. There was a higher rate of euploidy among blastocysts with higher quality grades in the NGS group than in the aCGH group for the trophectoderm (43.51\% vs $29.41 \%)$ and inner cell mass (59.11\% vs $25.00 \%)$.

Conclusion: Equivalent clinical findings were observed for aCGH and NGS for parental reciprocal chromosomal translocations. However, NGS has the potential to overcome the inherent limitations of aCGH, including the detection of mosaicism and smaller partial gains/losses, thereby providing improvements in the detection of euploid blastocysts, along with enhanced reliability and sensitivity.

\section{Background}

Reciprocal translocation is a chromosome structure rearrangement that occurs in approximately $0.2 \%$ unbiased new-born infants. It results in recurrent abortion with a prevalence of approximately $2.5-3.2 \%$ [1]. Reciprocal translocation carriers form a quadriradial chromosome during meiosis, resulting in the production of a large number of abnormal gametes. Consequent problems during gestation include early abortion, stillbirth, deformity, and mental retardation. Approximately $3 \%$ of couples with a history of subfertility are carriers of Robertsonian and reciprocal translocations. Preimplantation genetic testing (PGT) for chromosomal structural rearrangements is recommended for couples that are potential or known translocation carriers, and who desire to conceive a healthy child with their own gametes [2].

Theoretically, gamete abnormalities are mainly concentrated on chromosomes undergoing reciprocal translocation. Reciprocal translocation at an early stage is commonly detected using fluorescence in situ hybridization (FISH). FISH can test only a few chromosomes at a time. In the past few decades, several new technologies have been developed and refined, and can be applied to PGT. These include comparative genomic hybridization arrays (aCGH) and next-generation sequencing (NGS). They can test the 23 pairs of chromosomes simultaneously and can identify structural aberrations of chromosomes 
caused by reciprocal translocation. The aCGH technique combines chip and $\mathrm{CGH}$ technologies. It is widely used to detect DNA copy numbers according to the intensity ratio of two fluorescent signals produced at a resolution of 5-10 Mb and $1 \mathrm{Mb}$. NGS can detect copy numbers at the single-cell level to further test for chromosome mosaicism $[3,4]$. The tests focus on detection of abnormalities by screening for chromosome translocation.

Whether aCGH or NGS is a more suitable method for full-chromosome screening is unclear. To provide clarity, this retrospective analysis compared the results of aCGH and NGS to identify the more promising and cost-effective strategy for reciprocal and Robertsonian translocation carriers who wish to undergo PGT.

\section{Results}

Of the 272 biopsied embryos analysed, 58 blastocysts were subjected to aCGH analysis in 18 cycles and 214 blastocysts were subjected to NGS in 69 cycles. There were no significant differences in the mean maternal age, body mass index, basal follicle stimulating hormone, luteinizing hormone, prolactin, and oestradiol levels between the groups. The median number of retrieved, fertilized, and cleaved oocytes between groups did not differ significantly. The mean number of biopsied blastocysts and ratio of no embryo-transfer per cycle were also similar. The detailed data are summarized in Table 1.

[Table 1 near here]

The molecular karyotype was analysed in the cycles to identify reciprocal and Robertsonian translocations. Of the 58 blastular molecular karyotypes obtained from aCGH, $67.24 \%$ abnormal embryos were identified. They include $36.21 \%$ with a translocation-related abnormality, $17.24 \%$ with a nontranslocation-related abnormality, and $15.52 \%$ with a combination of abnormalities. Among the 214 blastocysts analysed by NGS, $59.81 \%$ of the embryos showed a chromosomal abnormality, including $28.50 \%$ due to parental-derived translocation, $13.08 \%$ with other abnormalities, and $18.22 \%$ with combined abnormalities. The ratios of euploidy and aneuploidy, including translocation-related, nontranslocation-related, and combined abnormalities were not significantly different between the aCGH and NGS groups (Table 2). In the NGS group, chromosomal abnormalities not related to translocations were reanalysed. Among the 39 chromosomes that were abnormal, $10.26 \%$ of the abnormalities occurred in chromosomes X, 16, and 7; followed by $7.69 \%$ abnormalities in chromosomes $10,6,2$, and $1 ; 5.13 \%$ abnormalities in chromosomes 21, 13, 11, 4, and 3; and 2.56\% abnormalities in chromosomes $Y, 17,12,9$, and 8 (Fig. 1). Among the 10 chromosomal abnormalities not related to translocations found in the aCGH group, $20 \%$ occurred in chromosome 4 , followed by $10 \%$ for chromosomes $X, 22,19,18,11,8$, and 3 (Fig. 2). The frequency of abnormalities in chromosome 4 was higher overall for both aCGH and NGS. 
Table 1

Basic parameters of samples used for aCGH and NGS testing.

\begin{tabular}{|llll|}
\hline Cycle parameters & aCGH & NGS & P-value \\
\hline Biopsied cycles & 18 & 69 & \\
\hline Maternal age (years) & $30.3 \pm 3.96$ & $29.04 \pm 4.10$ & 0.23 \\
\hline BMI (kg/m ${ }^{2}$ ) & $20.10 \pm 2.50$ & $21.50 \pm 2.91$ & 0.07 \\
\hline bFSH (IU/L) & $5.0 \pm 1.32$ & $5.82 \pm 1.38$ & 0.81 \\
\hline bLH (IU/L) & $4.28 \pm 2.47$ & $4.12 \pm 2.31$ & 0.80 \\
\hline PRL (IU/L) & $15.61 \pm 7.16$ & $17.54 \pm 7.83$ & 0.36 \\
\hline E2 (pg/mL) & $41.78 \pm 17.95$ & $41.28 \pm 16.89$ & 0.91 \\
\hline Duration of stimulation (days) & $11.06 \pm 1.59$ & $11.42 \pm 1.79$ & 0.43 \\
\hline Total gonadotropin doses (IU) & $2425.00 \pm 644.15$ & $2474.82 \pm 791.23$ & 0.81 \\
\hline Number of oocytes & $15.67 \pm 7.05$ & $15.09 \pm 6.61$ & 0.74 \\
\hline Number of metaphases II & $13.06 \pm 7.44$ & $12.75 \pm 5.78$ & 0.85 \\
\hline Number of fertilizations & $11.33 \pm 5.66$ & $11.54 \pm 5.38$ & 0.89 \\
\hline Number of cleavages & $10.17 \pm 5.70$ & $10.78 \pm 5.06$ & 0.66 \\
\hline Number of biopsied blastocysts & 58 & 214 & 0.32 \\
\hline Mean of biopsied blastocysts per cycle & $3.22 \pm 2.56$ & $3.10 \pm 1.96$ & 0.83 \\
\hline Number of biopsied blastocysts on day 5 & $1.94 \pm 2.1$ & $1.70 \pm 1.88$ & 0.63 \\
\hline Number of biopsied blastocysts on day 6 & $1.23 \pm 1.13$ & $1.41 \pm 1.19$ & 0.68 \\
\hline Proportion of balanced blastocysts (\%) & $32.76 \%(19 / 58)$ & $40.19 \%(86 / 214)$ & 0.30 \\
\hline Proportion of no embryo-transfer (\%) & $44.44 \%(8 / 18)$ & $31.88 \%(22 / 69)$ & 0.32 \\
\hline + Data are mean \pm standard deviation unless otherwise indicated. & & \\
\hline ₹ Abbreviations: aCGH, array comparative genomic hybridization; NGS, next-generation sequencing; \\
\hline BMI, body mass index; bFSH, basal follicle stimulating hormone;PRL, prolactin; E2, oestradiol \\
\hline
\end{tabular}


Table 2

Abnormal embryonic chromosomes determined by aCGH and NGS.

\begin{tabular}{|c|c|c|c|c|}
\hline & aCGH & NGS & $\begin{array}{l}\chi^{2} \\
\text { value }\end{array}$ & $\begin{array}{l}\mathrm{P}- \\
\text { value }\end{array}$ \\
\hline Total blastocysts & 58 & 214 & & \\
\hline Blastocysts of normal chromosomes & $\begin{array}{l}32.76 \% \\
(19 / 58)\end{array}$ & $\begin{array}{l}40.19 \% \\
(86 / 214)\end{array}$ & \multirow[t]{2}{*}{1.06} & \multirow[t]{2}{*}{0.30} \\
\hline Blastocysts of abnormal chromosomes & $\begin{array}{l}67.24 \% \\
(39 / 58)\end{array}$ & $\begin{array}{l}59.81 \% \\
(128 / 214)\end{array}$ & & \\
\hline $\begin{array}{l}\text { Blastocysts with translocation-related } \\
\text { abnormality }\end{array}$ & $\begin{array}{l}36.21 \% \\
(21 / 58)\end{array}$ & $\begin{array}{l}28.50 \% \\
(61 / 214)\end{array}$ & 1.29 & 0.26 \\
\hline Blastocysts without translocation-related & $\begin{array}{l}17.24 \% \\
(10 / 58)\end{array}$ & $\begin{array}{l}13.08 \% \\
(28 / 214)\end{array}$ & 0.66 & 0.42 \\
\hline Blastocysts with combined abnormality & $\begin{array}{l}15.52 \% \\
(9 / 58)\end{array}$ & $\begin{array}{l}18.22 \% \\
(39 / 214)\end{array}$ & 0.23 & 0.63 \\
\hline \multicolumn{5}{|c|}{ † Data are mean \pm standard deviation unless otherwise indicated } \\
\hline
\end{tabular}

[Table 2 near here]

[Figures 1 and 2 near here]

Maternal age was available for 272 blastocysts and permitted allocation to the $\geq 35$-years group $(n=31)$ and $<35$-years group $(n=241)$. The rate of chromosome abnormalities was higher in the $\geq 35$-years group than in the $<35$-years group for both methods, with no statistically significant difference $(P>0.05$, Table 3). Comparison of the rate of the normal blastocysts in samples from women $<35$-years old using aCGH and NGS revealed more normal blastocysts in the NGS group than in the aCGH group, but without statistical significance $(P=0.53)$. 
Table 3

Chromosome abnormalities in both maternal age groups.

\begin{tabular}{|c|c|c|c|c|c|c|}
\hline & \multicolumn{2}{|l|}{ aCGH } & \multicolumn{2}{|l|}{ NGS } & \multicolumn{2}{|l|}{ Total } \\
\hline $\begin{array}{l}\text { Maternal age } \\
\text { (years) }\end{array}$ & $\geq 35$ & $<35$ & $\geq 35$ & $<35$ & $\geq 35$ & $<35$ \\
\hline $\begin{array}{l}\text { Number of } \\
\text { blastocysts }\end{array}$ & 10 & 48 & 21 & 193 & 31 & 241 \\
\hline $\begin{array}{l}\text { Normal } \\
\text { blastocysts }\end{array}$ & $\begin{array}{l}20 \% \\
(2 / 10)\end{array}$ & $\begin{array}{l}35.41 \% \\
(17 / 48)\end{array}$ & $\begin{array}{l}38.10 \% \\
(8 / 21)\end{array}$ & $\begin{array}{l}40.41 \% \\
(78 / 193)\end{array}$ & $\begin{array}{l}32.26 \% \\
(10 / 31)\end{array}$ & $\begin{array}{l}39.41 \% \\
(95 / 241)\end{array}$ \\
\hline $\begin{array}{l}\text { Abnormal } \\
\text { blastocysts }\end{array}$ & $\begin{array}{l}80 \% \\
(8 / 10)\end{array}$ & $\begin{array}{l}64.58 \% \\
(31 / 48)\end{array}$ & $\begin{array}{l}61.90 \% \\
(13 / 21)\end{array}$ & $\begin{array}{l}59.59 \% \\
(115 / 193)\end{array}$ & $\begin{array}{l}67.74 \% \\
(21 / 31)\end{array}$ & $\begin{array}{l}60.58 \% \\
(146 / 241)\end{array}$ \\
\hline$x^{2}$ & 0.33 & & 0.04 & & 0.59 & \\
\hline P-value & 0.47 & & 0.83 & & 0.44 & \\
\hline
\end{tabular}

[Table 3 near here]

High-quality blastocysts were defined according to the combination of internal cell mass (ICM) or trophectoderm (TE) scores of A or B. Low-quality blastocysts had an ICM or TE score of C. A higher proportion of high-quality blastocysts had normal chromosomal patterns than aneuploidy, without statistical significance $(P=0.12$, Table 4$)$. In the NGS group, significantly more normal than abnormal embryos were observed among high-quality blastocysts $(P=0.03)$. There were more abnormal than normal chromosomes among high-quality embryos in the aCGH group $(P=0.22)$. There was a significant difference in the overall proportions of normal blastocysts among high-quality embryos detected using NGS and aCGH $(P=0.045)$. 
Table 4

Chromosome abnormalities with different qualities in aCGH and NGS.

\begin{tabular}{|c|c|c|c|c|c|}
\hline \multicolumn{2}{|c|}{ Chromosome constitution } & \multirow{2}{*}{$\begin{array}{l}\text { High-quality embryo } \\
25 \%(7 / 28)\end{array}$} & \multirow{2}{*}{$\begin{array}{l}\text { Low-quality embryo } \\
40 \%(12 / 30)\end{array}$} & \multirow{2}{*}{$\begin{array}{l}x^{2} \\
1.48\end{array}$} & \multirow{2}{*}{$\begin{array}{l}\text { P-value } \\
0.22\end{array}$} \\
\hline aCGH & normal & & & & \\
\hline & abnormal & $75 \%(21 / 28)$ & $60 \%(18 / 30)$ & & \\
\hline \multirow[t]{2}{*}{ NGS } & normal & $45.45 \%(65 / 143)$ & $29.58 \%(21 / 71)$ & \multirow[t]{2}{*}{4.98} & \multirow[t]{2}{*}{0.03} \\
\hline & abnormal & $54.55 \%(78 / 143)$ & $70.42 \%(50 / 71)$ & & \\
\hline \multirow[t]{2}{*}{ Total } & normal & $42.11 \%(72 / 171)$ & $32.67 \%(33 / 101)$ & \multirow[t]{2}{*}{2.38} & \multirow[t]{2}{*}{0.12} \\
\hline & abnormal & $57.89 \%(99 / 171)$ & $67.33 \%(68 / 101)$ & & \\
\hline \multicolumn{6}{|c|}{ † Data are mean \pm standard deviation unless otherwise indicated } \\
\hline
\end{tabular}

[Table 4 near here]

The TE scores of blastocysts reflects the formation of a cohesive epithelium. Thus, a cell with a higher TE grade has a greater chance of becoming a normal blastocyst. In the NGS group, decreased TE quality score was associated with an increase in the proportion of abnormal blastocysts, although the difference was not statistically significant (Table 5). No such tendency was observed in the aCGH group, in which there was a higher proportion of abnormal blastocysts associated with a higher TE grade, without a significant difference. Nevertheless, there were more blastocysts of higher TE grades ( $A$ and $B$ ) with normal chromosomes in the NGS group than in the aCGH group $(P=0.13)$ (Table 5$)$.

Table 5

Chromosome abnormalities with different trophectoderm score grades in aCGH and NGS.

\begin{tabular}{|c|c|c|c|c|c|}
\hline \multicolumn{2}{|c|}{ Chromosome constitution } & \multirow{2}{*}{$\begin{array}{l}\text { Grade A or B } \\
29.41 \%(10 / 34)\end{array}$} & \multirow{2}{*}{$\begin{array}{l}\text { Grade C } \\
37.5 \%(9 / 24)\end{array}$} & \multirow{2}{*}{$\begin{array}{l}\chi^{2} \\
0.42\end{array}$} & \multirow{2}{*}{$\begin{array}{l}\text { P-value } \\
0.52\end{array}$} \\
\hline $\mathrm{aCGH}$ & normal & & & & \\
\hline & abnormal & $70.58 \%(24 / 34)$ & $62.5 \%(15 / 24)$ & & \\
\hline \multirow[t]{2}{*}{ NGS } & normal & $43.51 \%(67 / 154)$ & $31.67 \%(19 / 60)$ & \multirow[t]{2}{*}{2.52} & \multirow[t]{2}{*}{0.11} \\
\hline & abnormal & $56.49 \%(87 / 154)$ & $68.33 \%(41 / 60)$ & & \\
\hline \multirow[t]{2}{*}{ Total } & normal & 73.33\% (77/188) & $26.67 \%(28 / 84)$ & \multirow[t]{2}{*}{1.42} & \multirow[t]{2}{*}{0.23} \\
\hline & abnormal & $66.47 \%(111 / 188)$ & $33.53 \%(56 / 84)$ & & \\
\hline
\end{tabular}

[Table 5 near here] 
Next, we analysed the relationship between ICM scores and chromosome abnormalities. A large majority of blastocysts were ICM grade A or B for both normal and abnormal embryos. In the NGS group, there was no difference in the rates of ICM grade $A$ or $B$ normal and abnormal blastocytes of $(P=0.53)$, whereas there were significantly more abnormal than normal embryos with grade $A$ or $B$ in the aCGH group $(P=0.06)$. In addition, there were significantly more normal embryos with ICM grade $A$ or $B$ in the NGS group than in the aCGH group $(P=0.05)$.

[Table 6 near here]

Table 6

Chromosome abnormalities with different inner cell mass scores in aCGH and NGS.

\begin{tabular}{|c|c|c|c|c|c|}
\hline \multicolumn{2}{|c|}{ Chromosome constitution } & \multirow{2}{*}{$\begin{array}{l}\text { Grade A or B } \\
25 \%(11 / 44)\end{array}$} & \multirow{2}{*}{$\begin{array}{l}\text { Grade C } \\
57.14 \%(8 / 14)\end{array}$} & \multirow{2}{*}{$\begin{array}{l}x^{2} \\
3.63\end{array}$} & \multirow{2}{*}{$\begin{array}{l}\text { P-value } \\
0.06\end{array}$} \\
\hline $\mathrm{aCGH}$ & normal & & & & \\
\hline & abnormal & $75 \%(33 / 44)$ & $42.86 \%(6 / 14)$ & & \\
\hline \multirow[t]{2}{*}{ NGS } & normal & $40.89 \%(83 / 203)$ & $27.27 \%(3 / 11)$ & \multirow[t]{2}{*}{0.34} & \multirow[t]{2}{*}{0.53} \\
\hline & abnormal & $59.11 \%(120 / 203)$ & $72.73 \%(8 / 11)$ & & \\
\hline \multirow[t]{2}{*}{ Total } & normal & $38.06 \%(94 / 247)$ & $44 \%(11 / 25)$ & \multirow[t]{2}{*}{0.34} & \multirow[t]{2}{*}{0.56} \\
\hline & abnormal & 61.94\% (153/247) & $56 \%(14 / 25)$ & & \\
\hline
\end{tabular}

\section{Discussion}

In this study, we retrospectively analysed the laboratory data on blastocyst cycles to identify balanced reciprocal and Robertsonian translocations using two different technologies: aCGH and NGS. Both methods have been validated to detect abnormalities effectively and simultaneously in all 24 human chromosomes $[3,5,6]$. However, their effectiveness has never been directly compared.

The clinical outcome of the two methods was the same, with no significant difference in the main parameters assessed, including the biopsied blastocysts per cycle, numbers of balanced blastocysts, and numbers of non-transferred embryos. Of the 272 blastocysts screened in a total of 95 cycles, the basal parameters and ovulation process in couples that included a partner harbouring a reciprocal translocation balances were not distinguished by either method. The accuracy and efficiency of aCGH and NGS appeared to be similar. There was $100 \%$ consistency in the detection of aneuploid embryos between aCGH and NGS, a well-established and highly validated method of aneuploidy screening in PGT cycles [7]. NGS detected normal and abnormal chromosomes with rates of $40.19 \%$ and $59.81 \%$, respectively. Of the abnormalities, $28.5 \%$ were related to translocation and $13.08 \%$ were not related to translocation and combined abnormalities. Similar results were obtained using aCGH. In general, couples in which one partner is a reciprocal translocation carrier produce varied numbers of unbalanced embryos 
depending on the location of the breakpoints [8]. We found an overall rate of $13.97 \%$ abnormalities ( $15.52 \%$ for aCGH and $13.08 \%$ for NGS) without no relation to translocation. A previous study found that $24 \%$ of embryos were aneuploid for other chromosomes with genetic balance for parental reciprocal translocation [8]. Therefore, neglecting the proportion of chromosomal abnormalities unrelated to translocations would erroneously reduce the overall rate of detection of abnormalities, which would ultimately affect the pregnancy outcome. This is the main reason NGS and aCGH technologies' detection of reciprocal translocations is considered superior to the conventional FISH method [9].

Concerning the distribution of chromosomes in blastocysts with abnormalities unrelated to translocation, most of the abnormalities were in chromosomes 7, 16, and X in the NGS group, and in chromosome 4 in the aCGH group. Chromosomal abnormalities are most frequently detected in chromosomes 16 and $\mathrm{X}$ in combined NGS and aCGH analyses [10]. This difference in the distribution of chromosomal abnormalities with and without translocations is a limitation of FISH technology for the diagnosis of structural chromosome abnormalities; an estimated $9.4 \%$ aneuploid embryos would not be detected [11]. NGS and aCGH can help decrease the risk of implantation failure or spontaneous abortions.

We further evaluated the relationship between different genetic testing platforms with the age-related frequency of aneuploidies; a maternal age of 35 years was the cut-off. We found no significant differences in the rates of normal and abnormal blastocysts screened in women younger or older than 35 years with either method. However, a significant increase in aneuploidy was previously observed in women > 39-years-of-age [12], and another study reported the lowest aneuploidy rate in women 26-37years-of-age, increasing to $33 \%$ at age 42 and $53 \%$ at age 44 [13]. In our study, only 10 blastocysts were analysed in women aged 35-40 years old, suggesting the possibility of bias. Considering all data from the PGT along with abnormalities of other chromosomes, there was a clear increase in the frequency of abnormalities for older women (data not shown). Interestingly, all women < 35-years-of-age had the same rate of normality using NGS and aCGH, indicating the appropriateness of both methods. The rates of single and dual abnormalities were $66.3 \%$ and $67.4 \%$, respectively, which is consistent with prior results [12]. Therefore, there are no substantial differences in NGS and aCGH for the detection of reciprocal translocation.

Since chromosomal abnormalities can manifest as abnormal embryo morphology, including changes in cell density, compaction, and degree of fragmentation [14], we also analysed the data concerning the effect of aneuploidy on the morphology of blastocysts in the cycles of reciprocal translocation testing using the two analytic techniques. As expected, there was a higher frequency of chromosomally normal blastocysts with a good morphology than with a poor morphology overall (68.57\% vs $31.43 \%)$. However, certain low-quality embryos displayed a normal molecular karyotype and high-quality blastocysts also had a high rate of abnormalities. These findings indicate a non-linear association between static morphological scoring and chromosomal status. These findings also agree well with the recent description that morphological selection was completely ineffective in avoiding aneuploidy to prevent an adverse pregnancy [15]. Another study demonstrated an unclear link between aneuploidy and morphology, with $52 \%$ embryos scoring within the top two grades being euploid, while $48 \%$ were 
abnormal [16]. We found a higher rate of abnormal blastocysts with Grade A or B to that of blastocysts of Grade $C$ for both TE (70.58\% vs $62.50 \%)$ and ICM (75\% vs. $42.86 \%)$ in the aCGH group. There was a much higher rate of normal blastocysts in higher-grade blastocysts in the NGS group. Considering the collective data, the results from NGS were more appropriate, suggesting a bias from the aCGH analysis, possibly due to the small sample of normal blastocysts in this analysis. Ideally, this comparison should be made with a larger dataset from multiple centres to generalize these findings [17]. Lai et al. [18] reported that with greater detection rates of segmental abnormalities, chromosomal gains and losses as small as 1.8$3 \mathrm{Mb}$ could be detected by NGS. Although mosaicism or small segments were detected by NGS, the results were classified as normal chromosome with aCGH, demonstrating that NGS is superior in detecting chromosomal abnormalities.

\section{Conclusion}

In conclusion, viable embryos were screened for chromosomal abnormalities using aCGH and NGS for couples in which one partner harboured a reciprocal translocation. Both methods showed a good clinical value in offering important counsel to the patients. The results of NGS were largely concordant with those of aCGH but should be further validated for diagnostics in PGT for chromosomal structural rearrangements. In summary, NGS is a more superior approach compared to aCGH as it has better detection of mosaicism and partial gains/losses. However, further large-scale and multicentre studies are required to confirm the reliability and efficacy of these methods.

\section{Methods}

\section{Data Collection and Samples}

The data of 272 embryos from 95 cycles were reviewed among couples. One partner had a balanced reciprocal translocation and had undergone PGT. There were 58 embryos in the aCGH group and 214 in the NGS group. Maternal age ranged from 20 to 40 years (mean $29.31 \pm 4.08$ years). Paternal age ranged from 24 to 50 years (mean $31.68 \pm 5.31$ years). The proportion of balanced reciprocal translocations in women < 35-years-old was 39.41\% (95/241), including 17 embryos in the aCGH group and 78 embryos in the NGS group. All biopsies were excised via laser punch and obtained at the blastocyst stage.

\section{Blastocyst Culture, Scoring, and Biopsy}

The quality of ICM was rated Grade A (tightly packed with many cells), Grade B (loosely grouped with several cells), and Grade C (few cells). TE quality was Grade A (many cells forming a cohesive layer), Grade B (few cells forming a loose epithelium), and Grade C (fewer large cells). All blastocysts were cultured in G2 medium (Vitrolife, Västra Frölunda, Sweden) and were biopsied using a ZILOS-tk laser punch (Hamilton Thorne Biosciences Inc., Beverly, MA, USA). Three to six TE cells were removed on day 5 or 6 of embryonic development. The biopsied cells were placed in $5 \mu \mathrm{L}$ buffer $(1 \times$ phosphate buffer for the aCGH group and $0.2 \mathrm{M} \mathrm{KOH}$ for the NGS group). The same protocols, including cell lysis and wholegenome amplification (WGA), were applied to the cells for the aCGH and NGS platforms. 


\section{aCGH Testing and NGS Analysis}

All cells from the TE of a blastocyte were subjected to aCGH microarray-based, 24-chromosome aneuploidy screening. WGA was conducted using the Gentisure Pre-screen Amplification kit (Agilent, Santa Clara, CA, USA) according to the manufacturer's instructions. The amplified DNA samples and reference DNAs supplied in the kit were labelled using Cy3 and Cy5 fluorescent-labelled nucleotides, respectively, also included in the kit. The labelled samples and reference DNAs were hybridized overnight in a 1:1 $2 \times$ Hi-RPM Hybridization Buffer and water mixture (Agilent) and applied to microarray slides (oligo aCGH/Chlp-on-chip Hybridization kit, Agilent). The slides were washed for $5 \mathrm{~min}$ in Buffer I at room temperature, followed by $1 \mathrm{~min}$ in Buffer $I I$ at $37^{\circ} \mathrm{C}$. The microarray slides were scanned and analysed using Agilent CytoGenomics software.

For NGS, the MALBAC single-cell WGA method was applied to amplify DNA, which was sequenced using a HiSeq 2500 platform (Illumina, San Diego, CA, USA), yielding two million reads per sample. The reads were analysed using a model YK001B kit (Yikon Genomics, Shanghai, China) and the sequencing libraries were constructed with NEBNext Ultra DNA kit (New England Biotechnology, Rowley, MA, USA). A 50\% increase or decrease in read counts reflected copy number gain or loss, respectively.

\section{Statistical analyses}

Statistical analysis was performed using the independent t-test and chi-square test. Data with P-values < 0.05 were considered statistically significant, with P-values $<0.001$ indicating statistically high significance.

\section{List of abbreviations}

aCGH, array comparative genomic hybridization; FISH, fluorescence in situ hybridization; ICM, internal cell mass; NGS, next-generation sequencing; PGT, preimplantation genetic testing; TE, trophectoderm

\section{Declarations}

\section{Declarations}

\section{Ethical approval}

The study was conducted under the approval of the ethics committee of Fujian Provincial Maternity and Children's Hospital, China.

\section{Consent for publication}

\section{Availability of data and materials}


The datasets used and/or analyzed during the current study are available from the corresponding author on reasonable request

\section{Competing interests}

The authors declare that they have no competing interests

\section{Funding}

The study was performed under the auspices of the Youth Project of Fujian Provincial Health Commission (Grant No. 2017117), Hospital Project of Fujian Maternal and Child Health Hospital (Grant No. 17-15) and National Nature Science Foundation of China (Grant No. 81803547).

\section{Authors' contribution}

ZB-H designed and conceptualized the project; DS-R, LY-H, SY, CQ-F, SJ-Q and HZ-Q collected samples and performed the experiments; DS-R drafted the manuscript. All authors read and approved the final manuscript.

\section{Acknowledgements}

We thanked all the colleagues for offering assistance to our study.

\section{References}

1. Ozawa $N$, Maruyama $T$, Nagashima $T$, Ono $M$, Arase $T$, Ishimoto $H$, et al. Pregnancy outcomes of reciprocal translocation carriers who have a history of repeated pregnancy loss. Fertil Steril. 2008;90:1301-4.

2. Otani T, Roche M, Mizuike M, Colls $P$, Escudero T, Munné S. Preimplantation genetic diagnosis significantly improves the pregnancy outcome of translocation carriers with a history of recurrent miscarriage and unsuccessful pregnancies. Reprod Biomed Online. 2006;13:869-74.

3. Fiorentino F, Spizzichino L, Bono S, Biricik A, Kokkali G, Rienzi L, et al. PGD for reciprocal and Robertsonian translocations using array comparative genomic hybridization. Hum Reprod. 2011;26:1925-35.

4. van Echten-Arends J, Mastenbroek S, Sikkema-Raddatz B, Korevaar JC, Heineman MJ, van der Veen $F$, et al. Chromosomal mosaicism in human preimplantation embryos: a systematic review. Hum Reprod Update. 2011;17:620-7. 
5. Schoolcraft WB, Treff NR, Stevens JM, Ferry K, Katz-Jaffe M. Scott RT Jr.

6. . Live birth outcome with trophectoderm biopsy, blastocyst vitrification, and single-nucleotide polymorphism microarray-based comprehensive chromosome screening in infertile patients. Fertil Steril. 2011;96:638-40.

7. Zhang C, Zhang C, Chen S, Yin X, Pan X, Lin G, et al. A single cell level based method for copy number variation analysis by low coverage massively parallel sequencing. PLoS One. 2013;8:e54236.

8. Fiorentino F, Biricik A, Bono S, Spizzichino L, Cotroneo E, Cottone G, et al. Development and validation of a next-generation sequencing-based protocol for 24-chromosome aneuploidy screening of embryos. Fertil Steril. 2014;101:1375-82.

9. Tobler KJ, Brezina PR, Benner AT, Du L, Xu X, Kearns WG. Two different microarray technologies for preimplantation genetic diagnosis and screening, due to reciprocal translocation imbalances, demonstrate equivalent euploidy and clinical pregnancy rates. J Assist Reprod Genet. 2014;31:84350 .

10. Lee VC, Chow JF, Lau EY, Yeung WS, Ho PC, Ng EH. Comparison between fluorescent in-situ hybridisation and array comparative genomic hybridisation in preimplantation genetic diagnosis in translocation carriers. Hong Kong Med J. 2015;21:16-22.

11. Shen J, Wu W, Gao C, Ochin H, Qu D, Xie J, et al. Chromosomal copy number analysis on chorionic villus samples from early spontaneous miscarriages by high throughput genetic technology. Mol Cytogenet. 2016;9:7.

12. Abdelhadi I, Colls P, Sandalinas M, Escudero T, Munné S. Preimplantation genetic diagnosis of numerical abnormalities for 13 chromosomes. Reprod Biomed Online. 2003;6:226-31.

13. Lukaszuk K, Liss J, Kunicki M, Jakiel G, Wasniewski T, Woclawek-Potocka I, et al. Anti-Müllerian hormone (AMH) is a strong predictor of live birth in women undergoing assisted reproductive technology. Reprod Biol. 2014;14:176-81.

14. Franasiak JM, Forman EJ, Hong KH, Werner MD, Upham KM, Treff NR, et al. The nature of aneuploidy with increasing age of the female partner: a review of 15,169 consecutive trophectoderm biopsies evaluated with comprehensive chromosomal screening. Fertil Steril. 2014;101:656-63.e1.

15. Stensen MH, Tanbo T, Storeng R, Byholm T, Fèdorcsak P. Routine morphological scoring systems in assisted reproduction treatment fail to reflect age-related impairment of oocyte and embryo quality. Reprod Biomed Online. 2010;21:118-25.

16. Fragouli E, Alfarawati S, Spath K, Wells D. Morphological and cytogenetic assessment of cleavage and blastocyst stage embryos. Mol Hum Reprod. 2014;20:117-26.

17. Alfarawati S, Fragouli E, Colls P, Stevens J, Gutiérrez-Mateo C, Schoolcraft WB, et al. The relationship between blastocyst morphology, chromosomal abnormality, and embryo gender. Fertil Steril. 2011;95:520-24.

18. Friedenthal J, Maxwell SM, Munné S, Kramer Y, McCulloh DH, McCaffrey C, et al. Next generation sequencing for preimplantation genetic screening improves pregnancy outcomes compared with 
array comparative genomic hybridization in single thawed euploid embryo transfer cycles. Fertil Steril. 2018;109:627-32.

19. Lai HH, Chuang TH, Wong LK, Lai HH, Chuang TH, Wong LK, et al. Identification of mosaic and segmental aneuploidies by next-generation sequencing in preimplantation genetic screening can improve clinical outcomes compared to array-comparative genomic hybridization. Mol Cytogenet. 2017;10:14.

\section{Figures}

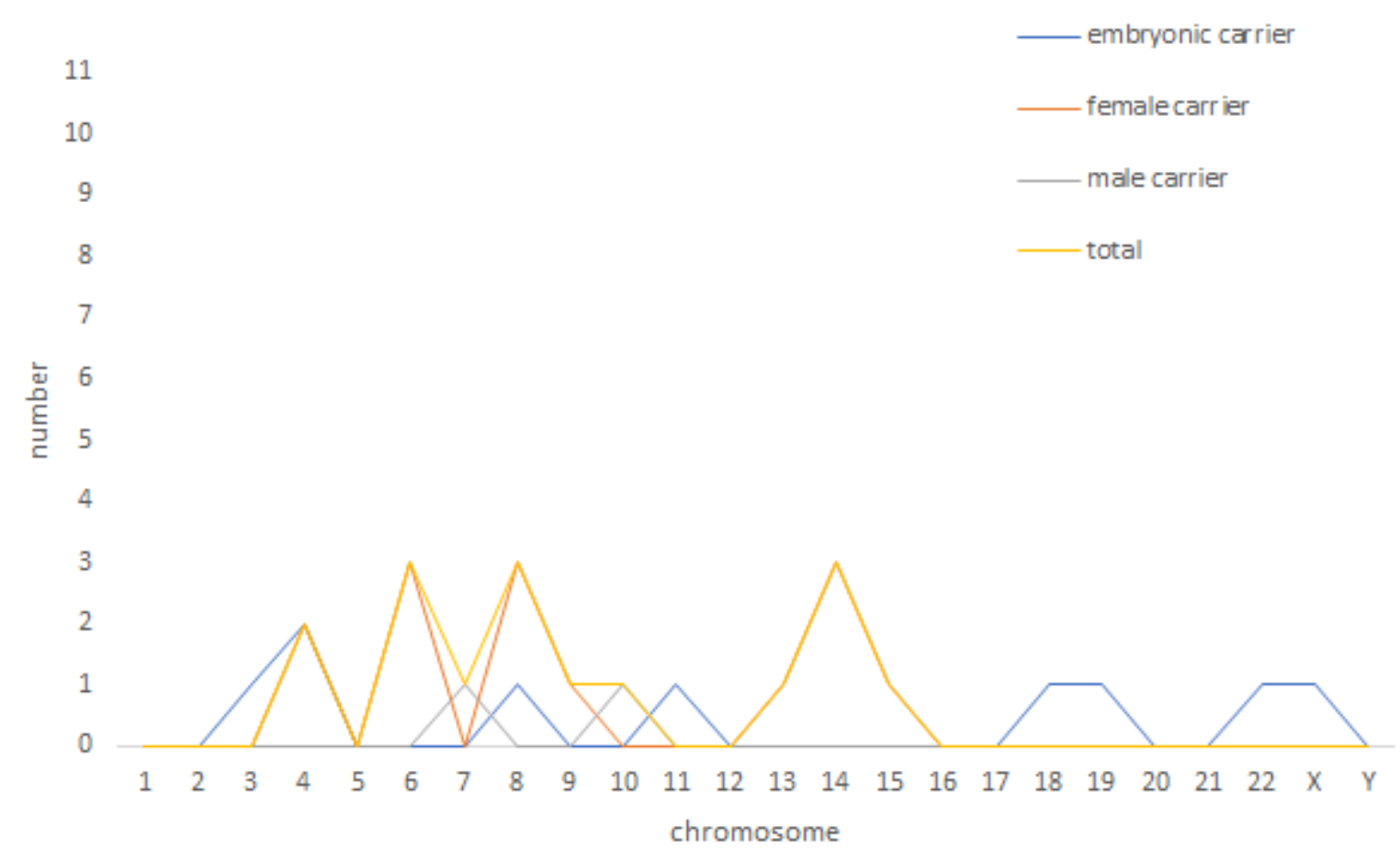

\section{Figure 1}

Distribution of abnormal chromosomes of blastocysts without a translocation-related abnormality in array comparative genomic hybridization. 


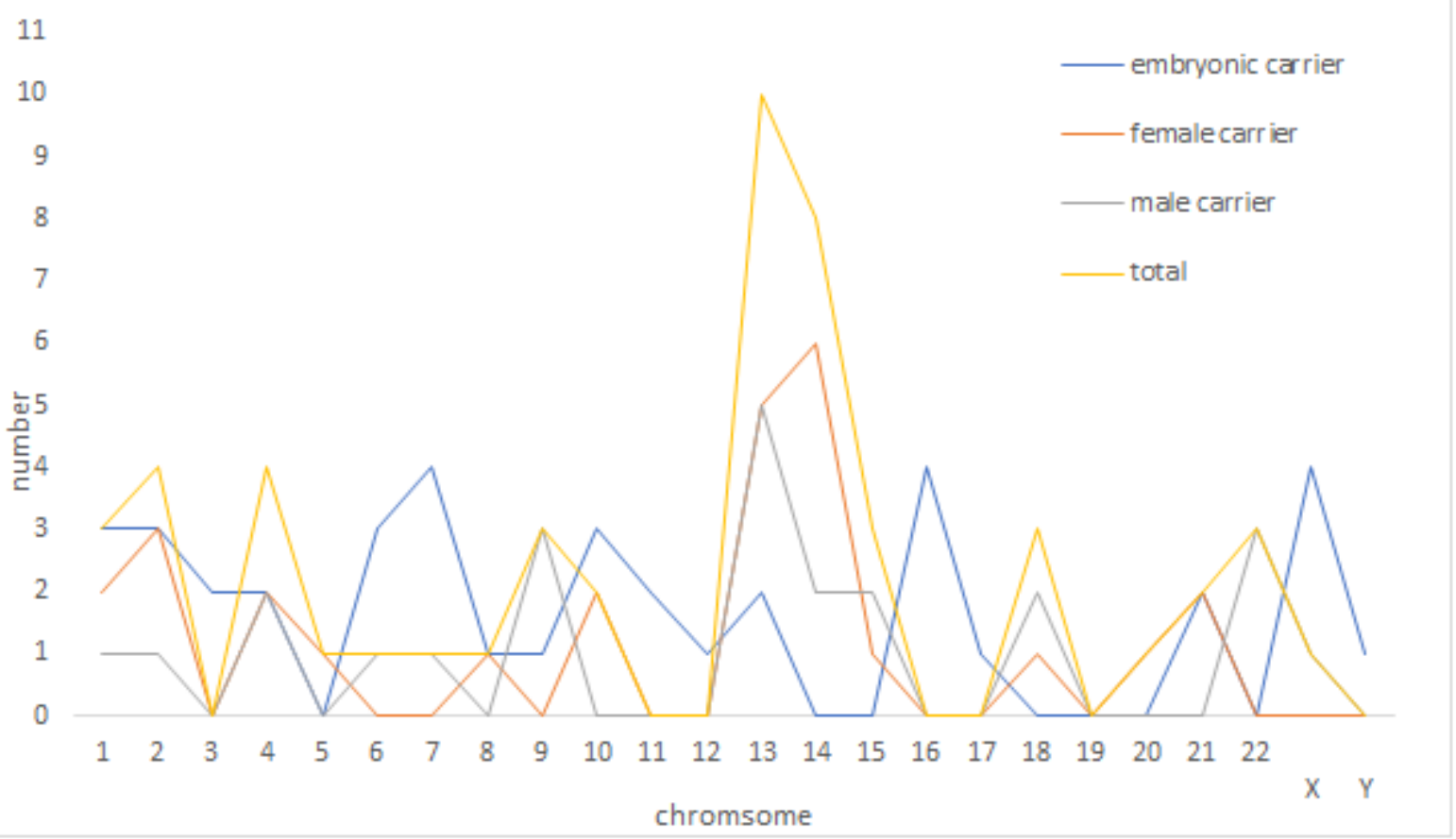

Figure 2

Distribution of abnormal chromosomes of blastocysts without translocation-related abnormalities in next-generation sequencing. 